\title{
A CMOS Mixed-Signal Interface with a RF Transmitter for a Micromachined Soil Moisture Sensor
}

\author{
Raul MORAIS ${ }^{1}$, Student Member, IEEE, A. VALENTE ${ }^{2}$, Student Member, IEEE, \\ J. H. CORREIA ${ }^{3}$, Member, IEEE, and C. COUTO ${ }^{4}$, Senior Member, IEEE \\ ${ }^{1,2}$ UTAD, DE, Quinta de Prados, Vila Real, Portugal, e-mail : (rmorais ${ }^{1}$, avalente $^{2}$ )@utad.pt \\ ${ }^{3,4}$ UM, DEI, Campus de Azurém, Guimarães , Portugal, e-mail: $\left(\right.$ jhc $^{3}$, ccouto ${ }^{4}$ )@ dei.uminho.pt
}

\begin{abstract}
This paper describes a CMOS mixed-signal interface with a RF transmitter. This die is assembled in a Multi-Chip-Module (MCM) micro-system together with the micromachined soil moisture sensor to achieve a cost-effective solution with accurate and reliable measurements for soil moisture in agriculture. The soil moisture probe, based on Dual-Probe Heat-Pulse (DPHP) method, is fabricated in bulkmicromachining technology. The DPHP method is based on the measurement of the maximum temperature rise at some distance from the heater, after applying a heat-pulse. The measurement of the temperature rise is obtained by subtracting soil temperature from the probe temperature. The mixed-signal interface is based on a pre-amplification stage and first-order sigma-delta modulator. The bit-stream output of the modulator is then applied to a counter as a first order decimation filter thus providing a 12-bit readout sample. Prior to transmission, data is encoded as a pulse width modulated (PWM) signal and then transmitted by means of an amplitude shift-keying (ASK) modulation. The transmitter features a VCO phase locked to the quartz crystal reference of $13.56 \mathrm{MHz}$ to achieve a carrier frequency of $433.92 \mathrm{MHz}$. A RF power amplifier based on class $\mathrm{E}$ topology was chosen. The CMOS mixed-signal interface with a RF transmitter has been implemented in a single-chip using a standard CMOS process (AMI $0.7 \mu \mathrm{m}$, n-well, 2 metals and 1 poly).
\end{abstract}

Index Terms-Wireless, Sigma-Delta, Soil-Moisture Sensors.

\section{INTRODUCTION}

In the field of environmental control applications such as irrigation management systems many sensors are needed for temperature, relative humidity, $\mathrm{CO}_{2}$ concentration, solar radiation and soil moisture.

The economics factors and the desire to minimize resource over-consumption have increased the requirements for irrigation management systems to manage water more efficiently. This resulted on a wider spectrum of available equipment for measuring soil water status [1].

Advances in silicon micromachining in the last years have encouraged a wide demand for silicon-based microsensors. Integrated microsensors, with on-chip interface circuitry, are currently replacing discrete sensors due to their inherent advantages, namely, low cost, accuracy, high reliability and onchip processing. To accomplish small, robust and inexpensive microsystems, it is desirable to integrate the soil moisture sensor with digital signal processing and wireless frontend. Therefore, this microsystem could be installed near plant roots for measuring real plant water needs. With wire0-7803-7912-8/03/\$17.00 @ 2003 IEEE

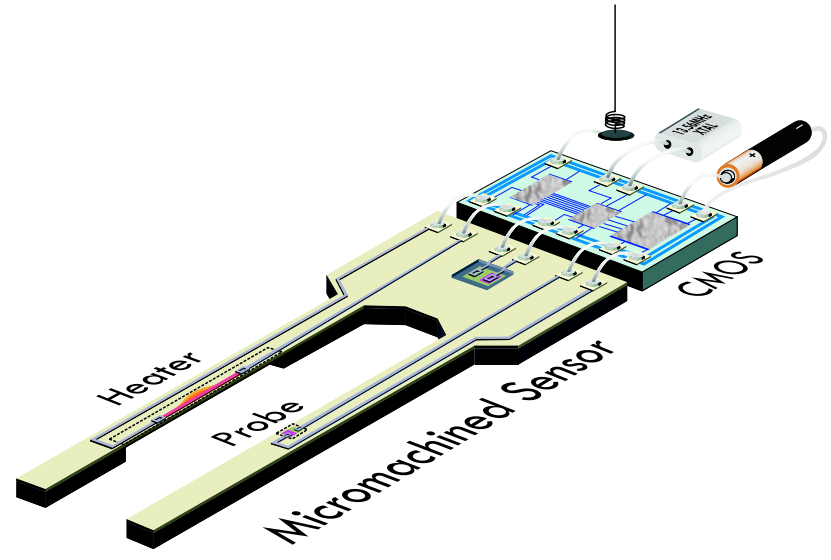

Fig. 1. System Overview

less capabilities, it would be possible to implement a smart irrigation control system to provide only the sufficient water, thus minimizing over-consumption.

\section{SYSTEM OVERVIEW}

The multi-chip module is basically made of two blocks, as depicted in Fig. 1: the micromachined sensor and the mixedsignal interface with a RF transmitter.

Since the bulk-micromachined sensor and electronics are implemented in different technologies, at this point the two system modules are being implemented in a multi-chip module. In the near future they will be implemented in only one chip using standard CMOS process with post-processing micromachining techniques.

\section{A. CMOS mixed-signal interface with $R F$ transmitter}

The implemented CMOS mixed-signal interface, outlined in Fig. 2, includes a pre-amplification stage, a first-order, continuous-time, fully-differential sigma-delta modulator, a counter as a first-order decimation filter, a shift register, and a RF transmitter. The carrier is generated on-chip by a frequency synthesizer.

The mixed-signal interface is responsible for controlling the heat pulse duration, analog-to-digital conversion, and data transmission. A 12-bit first-order sigma-delta converter performs the analog-to-digital conversion. Before transmission, data is encoded in a binary pulse width modulated signal, and afterwards the carrier of $433.92 \mathrm{MHz}$ is modulated 


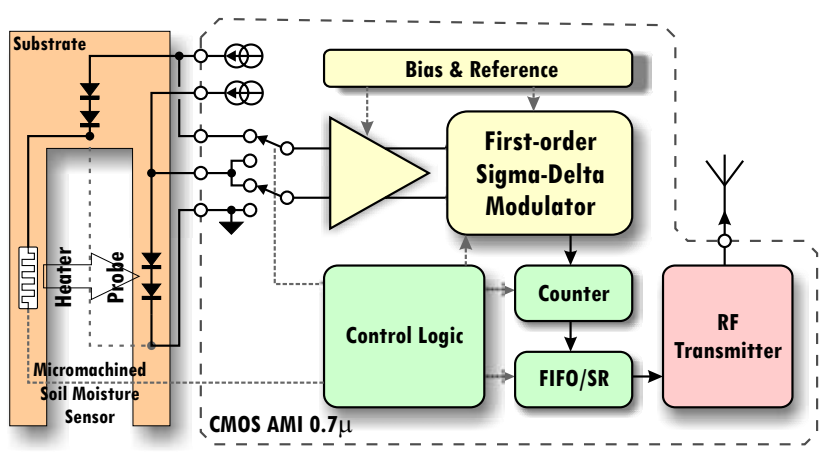

Fig. 2. Schematic representation of the mixed-signal interface

in ASK (Amplitude Shift Keying). A RF Power amplifier drives a small off-chip loop antenna.

The CMOS mixed-signal interface with a RF transmitter has been implemented in a single-chip using a standard CMOS process (AMI $0.7 \mu \mathrm{m}$, n-well, 2 metals and 1 poly).

\section{B. Micromachined Sensor Module}

The bulk-micromachined soil moisture sensor [2] is based on the dual-probe heat-pulse (DPHP) method to determine the volumetric heat capacity and hence the soil water content. Dimensions are $30 \mathrm{~mm}$ long, $6 \mathrm{~mm}$ wide and $0.8 \mathrm{~mm}$ high. The probe pitch is $3 \mathrm{~mm}$, allowing small-scale spatial measurements of water fractions of the soil, important for inferring soil moisture at plant root level.

The sensor consists of two needle probes mounted in parallel to provide a heater and a sensor probe. The heater uses polysilicon resistor for resistive heating (thermo resistive effect - Joule heat). The temperature sensors (probe and reference substrate) are made from micromachined $\mathrm{p}-\mathrm{n}$ junctions (Phosphorus - Boron).

The heat pulse is generated by applying a voltage to the heater for a fixed period of time. In the opposite rod, the maximum temperature rise above the substrate temperature (reference) is measured. Considering the expected range of water content ( $0 \%$ to $40 \%$ ), and that common sensitivity of integrated junction-based thermal sensors is approximately $-2.2 \mathrm{mV} /{ }^{\circ} \mathrm{C}$, then a $1 \%$ change in soil water content would cause a change in $\mathrm{p}-\mathrm{n}$ junction direct voltage drop of about $22 \mu V$ for each p-n junction.

\section{MiXed-Signal InTERfACE}

The maximum rise in temperature, $\Delta T_{m}$, is measured by subtracting the soil temperature signal from the substrate temperature (reference signal). This results in a very small signal with a full-scale of about $1 \mathrm{mV}$. The soil water contents must be measured with a resolution better than $1 \%$. This requirement leads to a merely $22 \mu \mathrm{V}$ sensor readout. Signal bandwidth is typically of the order of few Hz. This extremely small signal must be amplified with high gain to an appropriate signal level where it can be converted to the digital domain. The amplification of microvolt signals dictates the maximum amplifier offset allowed. To achieve an equivalent input offset of $10 \mu \mathrm{V}$ a chopper stabilization technique

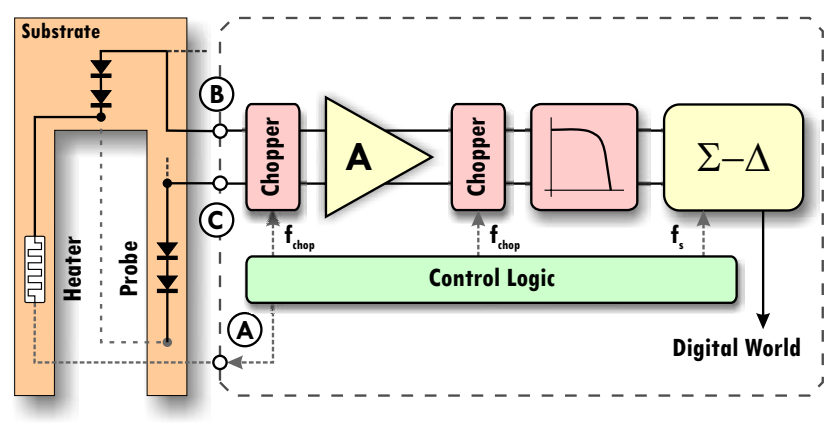

Fig. 3. Analog front-end

was employed [3]. The architecture of the mixed-signal interface is shown in Fig. 3, where $B$ represents the substrate temperature input (reference), $A$ the probe temperature input and $C$ the heater output control.

The analog front-end consists of a chopper modulator, a fully differential folded-cascode amplifier, a chopper demodulator and a low-pass filter. The input signal is modulated via a chopper modulator, which up-converts the signal to the chopping frequency. The modulated signal is then amplified and de-modulated back to the baseband. The amplifier input offset is by this way modulated at the chopping frequency and needs to be removed by filtering. The $1 / f$ noise is also removed if the chopping frequency is higher than the knee frequency. The low-pass filter is implemented with a second-order gm-c filter. In this application, the cut-off frequency of the filter was chosen as $500 \mathrm{~Hz}$. The chopping frequency has been set to $20 \mathrm{kHz}$, which is higher than the noise corner frequency. The chopper modulator, however, injects a residual offset in the signal due to non-idealities of the MOS switches, such as clock feedthrough and charge injection. A fully differential topology is used to improve noise immunity, reduce common-mode interference and minimize residual offset injected in the signal by the chopper modulators.

The amplified signal is now applied to a first-order SigmaDelta $(\Sigma-\Delta)$ converter, as depicted in Fig. 4. Sigma-Delta modulators trade bandwidth for resolution, which makes this architecture suitable for this application. In our case, having a very low-frequency signal $(<10 \mathrm{~Hz})$ and medium accuracy (12 bit) a one bit, first order $\Sigma-\Delta$ converter has been chosen because of the lower complexity, die area and power consumption when compared to higher order $\Sigma-\Delta$ converters. A further advantage of the $\Sigma-\Delta$ architecture is the relaxation of the component requirements used inside the loop. A high oversampling ratio of 512 was chosen to overcome the problems associated with low performance circuits.

However, this simpler architecture has severe drawbacks concerning pattern noise associated to DC inputs and low signal-to-noise ratio (SNR) [6]. DC stability and noise rejection are achieved here by switching the polarity of the differential input signal before the $\Sigma-\Delta$ converter. During half the conversion time, the modulator's output bit stream is applied to a counter (first order decimation filter). During the second half of the conversion time, the counter input is 


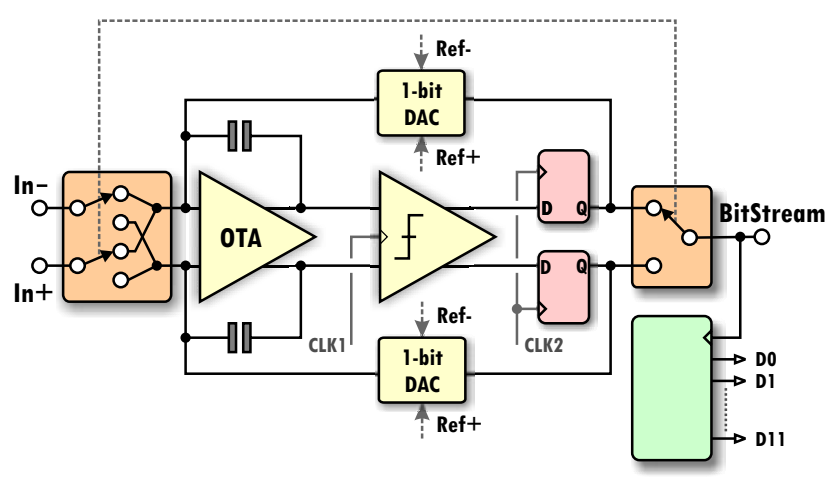

Fig. 4. First-order sigma-delta converter.

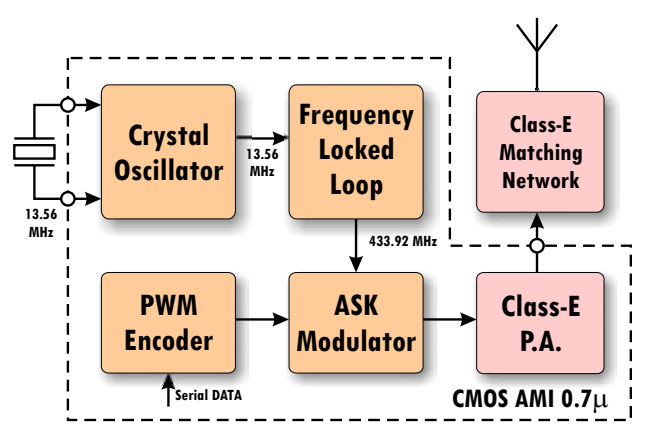

Fig. 5. RF transmitter diagram.

switched to the modulator's complementary output [4].

\section{RF Transmitter Module}

The RF transmitter module includes a crystal-controlled frequency synthesizer, a pulse-width modulation (PWM) encoding circuit, an Amplitude Shift Keying (ASK) modulator and a power amplifier as depicted in Fig. 5. For each sample, data is assembled together with a preamble for receiver synchronization, encoded and transmitted. Each packet is consisted by a 11-bit preamble, 12-bit data and 4-bit checksum.

One of the most important elements of the transmitter is the oscillator block. The oscillator should be capable of operating at a stable and predictable frequency over time. For this reason, the frequency synthesizer was implemented as a frequency-locked loop [5], using a $13.56 \mathrm{MHz}$ crystal reference oscillator to obtain the carrier frequency of $433.92 \mathrm{MHz}$.

Prior to transmission, data is encoded in pulse widths of $25 \%$ and $75 \%$ duty-cycle representing the logic levels ' 0 ' and ' 1 ' respectively, and then the PWM signal is transmitted by means of a amplitude shift keying modulation. The modulated signal is then applied to a class-E power amplifier that drives a small off-chip loop antenna to transmit the signal. In this first prototype, the typical class-E matching network is off-chip, as seen in Fig. 6. Since component values are suited for integration, they will be on-chip in the next prototype.

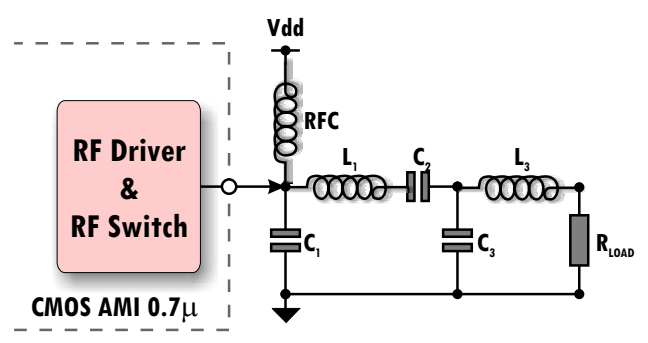

Fig. 6. Class-E power amplifier off-chip matching network.

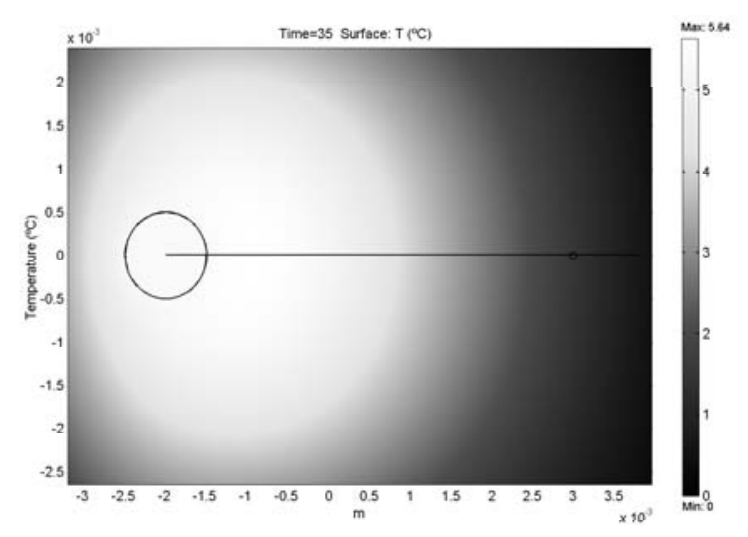

Fig. 7. Simulated $\triangle T_{m}$ distribution at $35 \mathrm{~s}$.

\section{RESUlts AND Discussion}

The micromachined sensor was simulated using FEMLAB. For this purpose, sensor rods are considered infinitely long and so the simulation was taken in a transversal section of the rod. Fig. 7 shows the temperature rise $\left(\triangle T_{m}\right)$ distribution $35 \mathrm{~s}$ after applying a heat-pulse of $q=600 \mathrm{Jm}^{-1}$ during $8 \mathrm{~s}$.

Soil samples of Almendra silt loam, which were wet to a predetermined water content and mixed, were packed into a cylinder $77 \mathrm{~mm}$ in diameter by $70 \mathrm{~mm}$ long, with the soil moisture sensor at the center. Measurements were taken and then the soil was weighed and dried at $105^{\circ} \mathrm{C}$ for $24 h$ to determine bulk density and water content (thermo-gravimetric method),

$$
\theta_{g}=\frac{(\text { wetweight })-(\text { dryweight })}{\text { dryweight }} .
$$

Table I lists the thermo-gravimetric soil water content $\left(\theta_{g}\right)$ and the measured values; maximum temperature rise $\left(\Delta T_{m}\right)$ and the heat applied per unit length of the line source $(q)$. The values of $\left(\theta_{v}\right)$ are calculated using,

$$
\theta_{v}=\frac{\frac{q}{e \pi r^{2} \triangle T_{m}}-\left(1.92 \times 10^{6} X_{m}+2.50 \times 10^{6} X_{o}\right)}{4.18 \times 10^{6}},
$$

where, $r, X_{m}$ and $X_{o}$ are the rod radius $(m)$, the mineral and organic fractions of soil respectively.

In soils with low organic matter, such as Almendra, $X_{o}$ is neglected. The value of $X_{m}$ is determined by dividing the soil bulk density by the particle density. An average value of $2.65 \mathrm{Mgm}^{-3}$ is often used for particle density of soils. Therefore, the calculated values of soil water content $\left(\theta_{v}\right)$ using Eq. 2, are in good agreement with thermo-gravimetric 
TABLE I

OBTAINED SOIL WATER CONTENTS.

\begin{tabular}{cccc}
\hline \hline$\theta_{g}\left(m^{3} m^{-3}\right)$ & $\Delta T_{m}\left({ }^{\circ} \mathrm{C}\right)$ & $q\left(J m^{-1}\right)$ & $\theta_{v}\left(m^{3} m^{-3}\right)$ \\
\hline 0 & 1.90 & 602 & 0 \\
0.1 & 1.35 & 605 & 0.106 \\
0.2 & 1.04 & 598 & 0.190 \\
0.3 & 0.85 & 601 & 0.308 \\
0.4 & 0.72 & 603 & 0.401 \\
\hline \hline
\end{tabular}

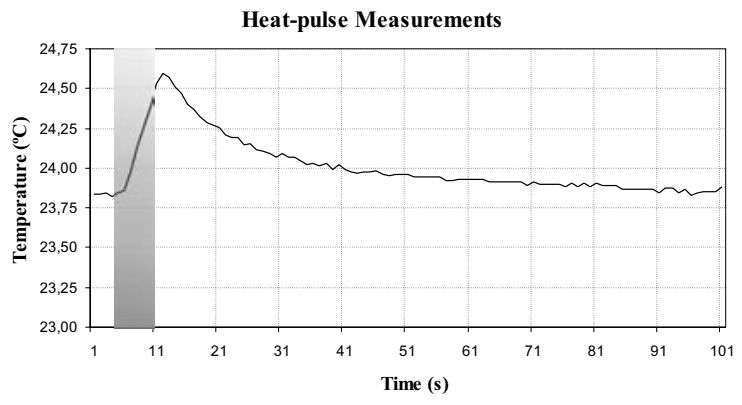

Fig. 8. Tipical Temperature by time data.

values. Fig. 8 shows a typical temperature response of heatpulse measurements. The shadow zone indicates the time of heat-pulse application.

Fig. 9 shows the simulated step response of the fully differential folded cascode amplifier for a $500 \mu \mathrm{V}$ input step and a gain of $66 \mathrm{~dB}$. Simulations have shown that it can be expected an effective resolution of 12-bit for the $\Sigma-\Delta$ modulator with a conversion time of $9.66 \mathrm{~ms}$ for a clock frequency of $423.75 \mathrm{kHz}$.

Fig. 10 shows a binary pattern ' $011^{\prime}$ PWM encoded signal (A) and transmitted signal (B) in a $50 \Omega$ load.

\section{Conclusions}

The design and modeling of a MCM-based micro-system for soil moisture measurements using the Dual-Probe HeatPulse (DPHP) method were achieved. The micro-system includes the soil moisture sensor, $\Sigma-\Delta$ converter, signal pro-

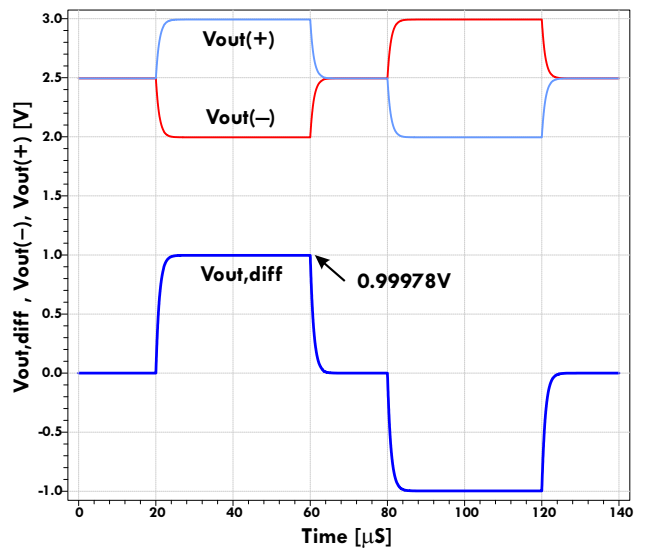

Fig. 9. Amplifier step response to $500 \mu V$ differential input.

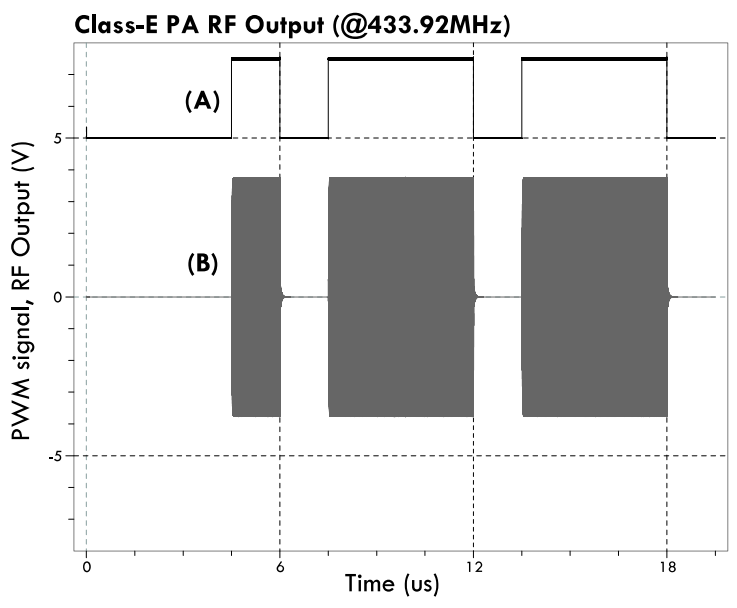

Fig. 10. RF Output

cessing circuits (digital filtering and sensor interface) and a $\mathrm{RF} 433.92 \mathrm{MHz}$ transmitter. The DPHP method showed to be the most appropriate to measure humidity at different soil depths, and therefore, close to plant roots in a non-destructive and automated manner. This is the first time that the DPHP method is implemented in a MCM-based micro-system and the first integrated sensor for soil moisture.

In this paper a CMOS mixed-signal interface with a RF transmitter for a micromachined soil moisture sensor has been proposed. Chip layout has been submitted to fabrication through Europractice IC service. A set of experiments has to be done for testing the reliability and precision of results.

Some enhancements are now being carried out, such as a receiver, among others. With bidirectional comunications capabilities, it will be possible to implement a wireless network of soil moisture sensors in a smart irrigation control system.

\section{REFERENCES}

[1] P. Charlesworth, Soil Water Monitoring, Irrigation Insights, 2000.

[2] A. Valente, C. Couto and J. H. Correia, "On-chip integrated silicon bulk-micromachined soil moisture sensor based on DPHP method," in Proceedings of Transducers'O1 and Eurosensors XV, 2001, pp.316319.

[3] C. Menolfi and Q. Huang, "A low-noise CMOS instrumentation amplifier for thermoelectric infrared detectors," IEEE Journal of Solid-State Circuits, vol.32, no.7, July, 1997, pp. 968-976

[4] Luca G. Fasoli, Frank R. Riedijk and Johan H. Huijsing, "A General Circuit for Resistive Bridge Sensors with Bitstream Output," IEEE Transactions on Instrumentation and Measurement, vol.46, no.4, April, 1997, pp. 954-960

[5] Rafael J. Betancourt-Zamora, Ali Hajimiri and Thomas H. Lee, "A $1.5 \mathrm{~mW}, 200 \mathrm{MHz}$ CMOS VCO for Wireless Biotelemetry," First International Workshop on Design of Mixed-Mode Integrated Circuits and Applications, July 28-30, 1997, Cancun, Mexico.

[6] J. C. Candy and G. C. Temes, Oversampling Delta-Sigma Converters, IEEE Press, New York: 1992, 1-25 